\title{
Clustering effects in fusion evaporation reactions with light even-even $\mathbf{N}=\mathbf{Z}$ nuclei. The ${ }^{24} \mathrm{Mg}$ and ${ }^{28} \mathrm{Si}$ cases
}

\author{
L. Morelli ${ }^{1, a}$, M. Bruno ${ }^{1}$, M. D'Agostino ${ }^{1}$, G. Baiocco ${ }^{2}$, F. Gulminelli ${ }^{3}$, M. Cinausero ${ }^{4}, M$. \\ Degerlier $^{5}$, D. Fabris ${ }^{6}$, F. Gramegna ${ }^{4}$, T. Marchi ${ }^{4}$, S. Barlini ${ }^{7}$, M. Bini ${ }^{7}$, G. Casini ${ }^{7}$, N. Gelli ${ }^{7}$, G. \\ Pasquali $^{7}$, S. Piantelli ${ }^{7}$, and S. Valdrè ${ }^{7}$. \\ ${ }^{1}$ Dipartimento di Fisica e Astronomia dell'Università and INFN, Sezione di Bologna, Bologna, Italy. \\ ${ }^{2}$ Dipartimento di Fisica dell'Università and INFN, sezione di Pavia, Pavia, Italy. \\ ${ }^{3}$ CNRS, UMR6534, LPC, Caen, France and ENSICAEN, UMR6534, LPC, Caen, France. \\ ${ }^{4}$ INFN, Laboratori Nazionali di Legnaro, Legnaro (Padova), Italy. \\ ${ }^{5}$ University of Nevsehir, Science and Art Faculty, Physics Department, Nevsehir, Turkey. \\ ${ }^{6}$ INFN, Sezione di Padova,, Padova, Italy. \\ ${ }^{7}$ Dipartimento di Fisica dell'Università and INFN, Sezione di Firenze, Firenze, Italy.
}

\begin{abstract}
.
In the recent years, cluster structures have been evidenced in many ground and excited states of light nuclei $[1,2]$. The decay of highly excited states of ${ }^{24} \mathrm{Mg}$ is studied in fusion evaporation events completely detected in charge in the reactions ${ }^{12} \mathrm{C}+{ }^{12} \mathrm{C}$ and ${ }^{14} \mathrm{~N}+{ }^{10} \mathrm{~B}$ at 95 and $80 \mathrm{MeV}$ incident energy, respectively, and compared to the results of a pure statistical model [3, 4].

Inclusive variables are in general well reproduced by the model. We found clear deviations from the statistical model if we select emission channels involving multiple $\alpha$ particles which are more probable than expected from a purely statistical behavior. Data from ${ }^{12} \mathrm{C}+{ }^{12} \mathrm{C}$ reaction have been analyzed in order to study the decay of the Hoyle state of ${ }^{12} \mathrm{C}^{*}$ with two different selections: peripheral binary collisions and $6 \alpha$ decay channel in central events. To continue the investigation on light systems, we have recently measured the ${ }^{16} \mathrm{O}+{ }^{12} \mathrm{C}$ reaction at three different beam energies, namely $\mathrm{E}_{\text {beam }}=90,110$ and $130 \mathrm{MeV}$. Preliminary results are presented.
\end{abstract}

\section{INTRODUCTION}

The NUCL-EX collaboration has recently started an experimental campaign of exclusive measurements of fusion-evaporation reactions with light nuclei as interacting partners. The aim is to progress in the understanding of statistical properties of the decay of light nuclei at excitation energies above particle emission thresholds and to measure observables linked to the presence of cluster structures in nuclear excited levels.

The statistical decay of hot nuclei and nuclear clustering are very active research topics in nuclear physics [1]. Using the statistical theory of Compound Nucleus (CN) decay, the detailed output of a fusion-evaporation reaction is uniquely predicted under the knowledge of nuclear ground state

a e-mail: luca.morelli@bo.infn.it 
properties and level densities. The knowledge of level densities is not only important for the understanding of nuclear structure [5], but it is also required for different applications of nuclear physics, from nucleosynthesis calculations to reactor science.

Despite the interest of this issue, few studies, mainly based on inclusive experiments, exist on the evaporation of very light nuclei $\left(\mathrm{A} \sim 20\right.$ region) at relatively high excitation energy $\left(\varepsilon^{*} \approx 3 \mathrm{~A} . \mathrm{MeV}\right)$. The interest on this mass - excitation energy region is easily justified: from the experimental point of view, measuring light nuclei means low multiplicity events, which, together with a high detection coverage and high energy and angular resolution, leads to the possibility of achieving a complete event reconstruction, thus having a full control on the reaction mechanism.

In addition, the knowledge of the decay mechanism could help in the backtracing procedure in multifragmentation events. Indeed in the statistical framework fragments formed in the freeze-out volume are mainly in this energy and mass region.

Nuclear structure signatures are especially evident in light nuclei, even at high excitation energy. By using an exclusive channel selection and a highly constrained statistical code, it is possible to put into evidence deviations from a statistical behavior in the decay of the hot sources formed in the collision. Indeed some excited states of different nuclei in this mass region are known to present pronounced cluster structures. These correlations may persist in the ground state along some selected isotopic chains [6] and, according to the Ikeda diagrams [2], $\alpha$-clustered excited states are sizeably expected at high excitation energies close to the multi-alpha decay threshold in all even-even $\mathrm{N}=\mathrm{Z}$ nuclei.

The subject of $\alpha$-clustering has been a central issue in nuclear physics and has witnessed a gain of interest in recent years. On the theoretical side, highly sophisticated ab-initio calculations have shown pronounced cluster features in the ground state of a large number of light nuclei [6]. Concerning experimental research, rotational bands consistent with $\alpha$-cluster structures have been identified in different even-even light nuclei and shown to persist even along their isotopic chains. Such effects can be experimentally seen as an excess of cluster production with respect to the prediction of the statistical model, provided that the ingredients of the latter are sufficiently constrained via experimental data. A campaign of exclusive measurements of fusion-evaporation reactions with light nuclei as interacting partners is currently carried on by our collaboration. In the framework of this campaign, the ${ }^{12} \mathrm{C}+{ }^{12} \mathrm{C}$ and ${ }^{14} \mathrm{~N}+{ }^{10} \mathrm{~B}$ reactions have been measured in order to study the decay of the same ${ }^{24} \mathrm{Mg}$ compound nucleus, populated at the same excitation energy but through different entrance channels. The results obtained in our first measurements are briefly reported in the following sections.

A very interesting clustering effect is for instance that of the so-called Hoyle state, i.e. the excited state $0^{+}$at $7.65 \mathrm{MeV}$ of ${ }^{12} \mathrm{C}$, which plays a decisive role in stellar nucleosynthesis of ${ }^{12} \mathrm{C}$.

Recently different experiments have studied the low-lying ${ }^{12} \mathrm{C}$ excited states and in particular the Hoyle state at $7.65 \mathrm{MeV}$ excitation energy [7] and several interesting features of these states have been pointed out [8-14].

In several cases experimental results have interpreted the Hoyle state as mainly sequential through an intermediate ${ }^{8} \mathrm{Be}_{g s}$ decay [8-12]; other experimental data show sizeable amount of instantaneous three $\alpha$-particle decay $[13,14]$ where the three $\alpha$ particles are characterized by low kinetic energies and low kinetic energy dispersion. Most of the results indicate a nearly complete agreement with a sequential decay with a very small amount $(0.2 \%$ [12]) of the instantaneous one.

In this work we show some preliminary results from the analysis of semiperipheral ${ }^{12} \mathrm{C}+{ }^{12} \mathrm{C}$ reactions where the projectile decays in three $\alpha$-particles passing through the Hoyle state.

The results compatible with indications for non-sequential decay have been obtained in reactions involving heavy ions $[13,14]$. We have therefore investigated the Hoyle state when obtained in the 
decay chain of ${ }^{24} \mathrm{Mg}^{*}$ formed in central collisions and in particular we have analyzed six $\alpha$-particles in the final stage of the ${ }^{24} \mathrm{Mg}^{*}$ decay.

We will compare the experimental results both for central and peripheral collisions with the prediction of a model based on Hauser-Feshbach formalism which includes light nuclei ecxcited states. The main features of this model have been extensively described in [4]. The predictions have been filtered in order to be compared to experimental data, taking into account detector characteristics such as thresholds, energy and angular resolutions. Data have been also compared to filtered simple model calculations for simultaneous decay (DDE), i.e. three $\alpha$-particles simultaneously emitted from the excited Carbon and for a linear chain (DDL), i.e. two $\alpha$-particles emitted back to back with the third $\alpha$-particle at rest in the ${ }^{12} \mathrm{C}$ frame.

Similary to the ${ }^{24} \mathrm{Mg}^{*}$, the compound nucleus ${ }^{28} \mathrm{Si}^{*}$ can be formed through fusion of $\alpha$-cluster stable nuclei in the entrance channel and in the future of light radioactive beams provided by SPES. It is interesting for such systems that they can be studied at different beam energies, thus extracting information on how (and to what extent) structure effects are still at play in the decay of hot nuclei at different excitation regimes.

\section{THE EXPERIMENTS}

The experiments were performed at the LNL (Laboratori Nazionali di Legnaro), with ${ }^{12} \mathrm{C},{ }^{14} \mathrm{~N}$ and ${ }^{16} \mathrm{O}$ beams provided by the XTU TANDEM accelerator. We have studied the two systems ${ }^{12} \mathrm{C}+{ }^{12} \mathrm{C}$ and ${ }^{14} \mathrm{~N}+{ }^{10} \mathrm{~B}$, leading to the same compound nucleus ${ }^{24} \mathrm{Mg} *$ at the same excitation energy. The decay of the ${ }^{28} \mathrm{Si}^{*}$ compound nucleus has been investigated using ${ }^{16} \mathrm{O}+{ }^{12} \mathrm{C}$ reaction at three different energies. For these last reactions the analysis has been focused up to now on the necessary preliminary checks on the quality of collected data. Charge (and mass, where the information is available) identification and energy calibration are in progress.

The apparatus has been described in [15]. Here we recall the main features. The experimental setup is composed of the GARFIELD apparatus and the Ring-Counter (RCo) annular detector, fully equipped with digital electronics [16]. The forward polar angles $\left(7^{\circ} \div 17^{\circ}\right)$ are covered by a three stage apparatus ( $\mathrm{RCo}$ ), ionization chamber (IC), silicon strip s ( $\mathrm{Si}$ ) and $\mathrm{CsI}(\mathrm{Tl})$ scintillators. The angular resolution is $\sim \pm 0.7^{\circ}$ for the polar angle and $\sim \pm 11^{\circ}$ for the azimuthal one for particles detected in the scintillators. The performances of the RCo allow to reach energy determination with the accuracy of percent, charge identification of particles and fragment with threshold as low as $0.8 \div 1 \mathrm{AMeV}$ and mass identification of light isotopes with $\sim 6 \mathrm{AMeV}$ threshold through $\triangle \mathrm{E}-\mathrm{E}$ technique in Si$\mathrm{CsI}(\mathrm{Tl})$ and/or pulse shape analysis (PSA)in the CsI [15]. The remaning polar angles $\left(\theta=30^{\circ} \div 170^{\circ}\right)$ are covered by a two stage, drift ionization chamber - CsI(Tl) scintillator apparatus (GARFIELD). Energy is determined with accuracy of the order of few percent and reaction products are identified in charge and mass (for light products) with a threshold around $1 \mathrm{AMeV}$ [15]. The combination of the two devices allows a nearly- $4 \pi$ coverage, which, combined with a high granularity, permits to measure the charge, the energy and the emission angles of nearly all the charged reaction products, with an excellent discrimination of the different reaction mechanisms.

\section{RESULTS}

\subsection{Central ${ }^{12} \mathrm{C}+{ }^{12} \mathrm{C}$ and ${ }^{14} \mathrm{~N}+{ }^{10} \mathrm{~B}$ reaction}

The selection of the fusion-evaporation mechanism is based on the coincidence between LCP's and a fragment detected at forward angles ( $\mathrm{RCo}$ ). At the same time we ask the complete charge detection and longitudinal momentum conservation. 

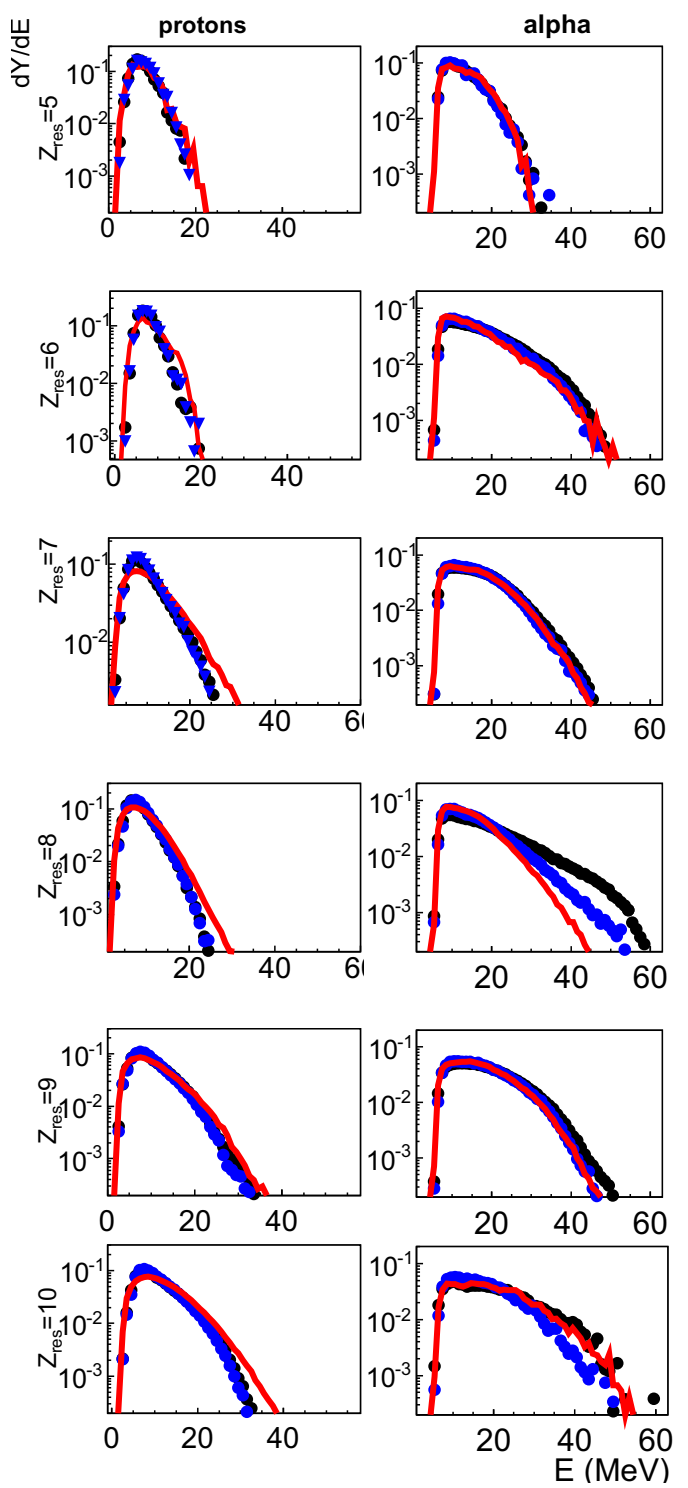

Figure 1. Proton (left part) and $\alpha$ (right part) energy spectra in complete $\mathrm{Z}_{d e t}=12$ events detected in coincidence with a residue of charge $Z_{r e s}$, indicated in each figure column. Data (black and blue for ${ }^{12} \mathrm{C}+{ }^{12} \mathrm{C}$ and ${ }^{14} \mathrm{~N}+{ }^{10} \mathrm{~B}$ respectively) are compared to model calculations (lines) for ${ }^{12} \mathrm{C}+{ }^{12} \mathrm{C}$ (see text for details). All distributions are normalized to unitary area.

We compare experimental data to the predictions of a dedicated Monte Carlo Hauser-Feshbach (HF $\ell$ ) code for the decay of the compound nucleus [3, 4], explicitly including all the experimentally measured particle unstable levels from the archive NUDAT2(http://www.nndc.bnl.gov/nudat2/). For both reactions the best reproduction of the systematics of the fusion cross sections is obtained assuming two different maximum values $\left(\mathrm{J}_{0 \max }\right)$ for the angular momentum distribution of the hot fused 
source [17]. In particular we use $\mathrm{J}_{0 \max }=18 \hbar$ for ${ }^{12} \mathrm{C}+{ }^{12} \mathrm{C}$ reaction and $\mathrm{J}_{0 \max }=15 \hbar$ for ${ }^{14} \mathrm{~N}+{ }^{10} \mathrm{~B}$ reaction and we adopt the same diffusness parameter $\Delta J=2$ for both reactions. Energy spectra for protons and $\alpha$ particles detected at GARFIELD angles are plotted in figure 1, for residues of different charge, for the ${ }^{14} \mathrm{~N}$ reaction, and compared to $\mathrm{HF} \ell$ [4] calculations and to data for the ${ }^{12} \mathrm{C}+{ }^{12} \mathrm{C}$ reaction. For both reactions, a good reproduction of proton and $\alpha$ energy spectra is achieved in all channels. Some discrepancies are present in events with an Oxygen residue, where the energy tails for $\alpha$ particles are not reproduced by the model, especially for the ${ }^{12} \mathrm{C}+{ }^{12} \mathrm{C}$ case. It has been already shown in [18] that this discrepancy is mostly due to the an extra experimental cross section for channels of the type ( $2 \alpha$, $\left.{ }^{16} \mathrm{O}^{g s / *}\right)^{1}$. Such outgoing channels populated in the ${ }^{12} \mathrm{C}+{ }^{12} \mathrm{C}$ collisions can be attributed to an entrance channel effect, given the $\alpha$-like structure of reaction partners and produced fragments. Indeed, for the ${ }^{14} \mathrm{~N}+{ }^{10} \mathrm{~B}$ case inclusive distributions are better reproduced by calculations. This seems not to exhaust the total discrepancy with $\mathrm{HF} \ell$ calculations, as we infer from the energy spectrum of $\alpha$ particles in coincidence with Oxygen and with ${ }^{20} \mathrm{Ne}$ for the ${ }^{14} \mathrm{~N}$ reaction.

Thanks to the completeness of event reconstruction, an estimate of the dissipated energy [18] can be extracted to further investigate all the channels involving alpha particles in both reactions: $Q_{k i n}=\sum_{i=1}^{2} E_{\alpha_{i}}+E_{Z_{\text {Res }}}-E_{\text {beam }}$, where $E_{\alpha_{i}}$ and $E_{Z_{\text {Res }}}$ are, respectively, the laboratory energy of $\alpha$ particles and residues, and $E_{\text {beam }}$ is the energy of the incident projectile. Figure 2 displays the obtained $Q_{k i n}$ distributions for ${ }^{12} \mathrm{C}+{ }^{12} \mathrm{C}$ (black dots) and ${ }^{14} \mathrm{~N}+{ }^{10} \mathrm{~B}$ (blue dots) reactions, taking into account the different initial Q-value for the two reactions. In particular we plot only the channels with the maximum $\alpha$ multiplicity associated to the residue of charge $\mathrm{Z}$.
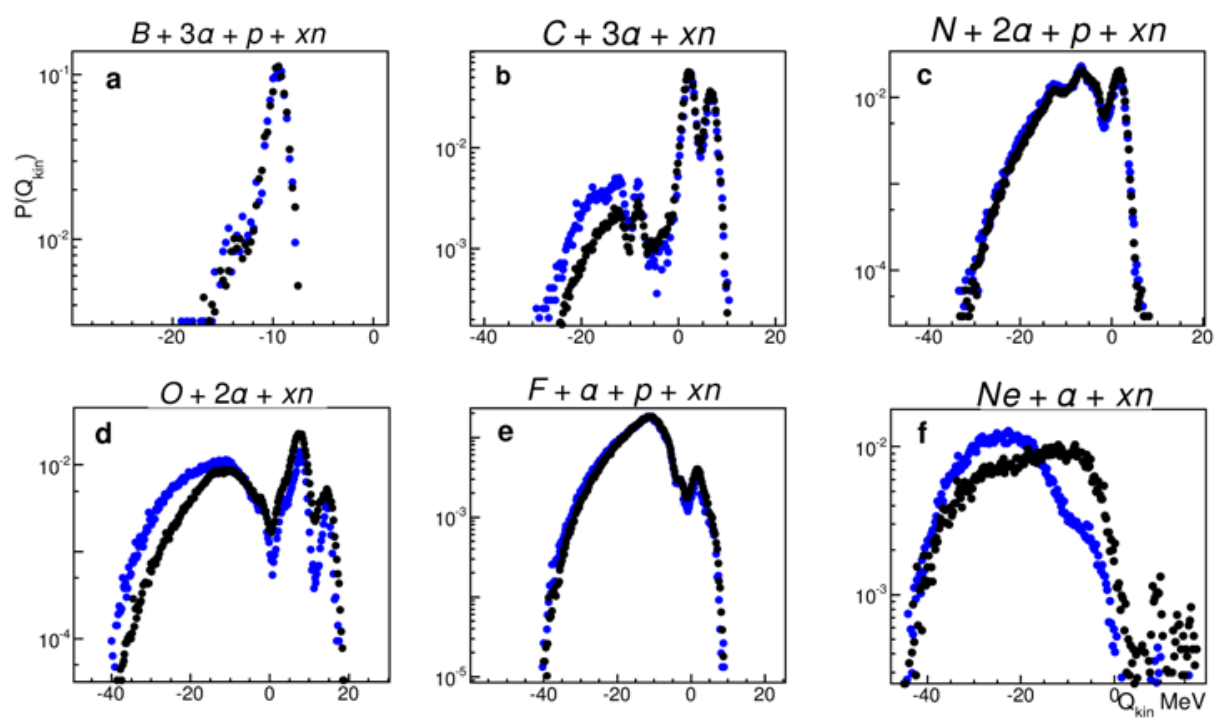

Figure 2. $\mathrm{Q}_{k i n}$-values distribution when the residue of charge from 5 to 10 , indicated in each panel, is detected in coincidence with the maximum possible number of $\alpha$-particles. Data are from ${ }^{12} \mathrm{C}+{ }^{12} \mathrm{C}$ (black dots) and from ${ }^{14} \mathrm{~N}+{ }^{10} \mathrm{~B}$ (blue dots) reactions. The $\mathrm{Q}_{\text {kin }}$ is normalized to the $\mathrm{Q}$-value of ${ }^{14} \mathrm{~N}+{ }^{10} \mathrm{~B}$ data. For more details see text.

In the $Q_{k i n}$ distribution for the channel $\left(2 \alpha,{ }^{16} \mathrm{O}^{g s / *}\right.$ figure $\left.2 \mathrm{~d}\right)$, the two peaks correspond to $\alpha$-decay chains, starting from the ${ }^{24} \mathrm{Mg}^{*}$ compound nucleus and leaving a ${ }^{16} \mathrm{O}$ residue either in its ground state

${ }^{1} \mathrm{O}^{g s / *}$ indicate the ${ }^{16} \mathrm{O}$ either in the ground or in an excited state 
Table 1. Most probable experimental channel and its branching ratio together with the value predicted by the $\mathrm{HF} \ell$ calculations. Errors on the experimental value (about 5\%) take into account both statistical error and the possible particle contamination

\begin{tabular}{|l|l|l|l|l|l|}
\hline$Z_{\text {res }}$ & Channel & $B R_{\text {exp }}$ NB (\%) & $B R_{H F \ell} \mathrm{NB}(\%)$ & $B R_{\text {exp }}$ CC (\%) & $B R_{H F \ell}$ CC (\%) \\
\hline 5 & ${ }^{11-x n} \mathrm{~B}+3 \alpha+\mathrm{p}+\mathrm{xn}$ & 99 & 100 & 99 & 100 \\
\hline 6 & ${ }^{12-x n} \mathrm{C}+3 \alpha+\mathrm{xn}$ & 96 & 74 & 98 & 78 \\
\hline 7 & ${ }^{15-x n} \mathrm{~N}+2 \alpha+\mathrm{p}+\mathrm{xn}$ & 90 & 95 & 91 & 95 \\
\hline 8 & ${ }^{16-x n} \mathrm{O}+2 \alpha+\mathrm{xn}$ & 56 & 15 & 63 & 15 \\
\hline 9 & ${ }^{19-x n} \mathrm{~F}+\alpha+\mathrm{p}+\mathrm{xn}$ & 91 & 93 & 92 & 88 \\
\hline 10 & ${ }^{22-x n} \mathrm{Ne}+\alpha+\mathrm{nx}$ & 47 & 3 & 26 & 3 \\
\hline
\end{tabular}

or in one of its excited bound states. The Q-values $Q_{k i n}=-15.78 \mathrm{MeV}$ for ${ }^{12} \mathrm{C}$ and $Q_{k i n}=-0.8$ $\mathrm{MeV}$ for ${ }^{14} \mathrm{~N}$ reactions correspond to the opening of the 4-body channel ${ }^{15} \mathrm{O}+n+\alpha+\alpha$. Neutrons are not detected in the experiments, and the broader distribution observed for lower $Q_{k i n}$ values is due to events in which neutron(s) emission has taken place. A difference in the relative population of less dissipative events is evident between the ${ }^{12} \mathrm{C}+{ }^{12} \mathrm{C}$ (black dots) and ${ }^{14} \mathrm{~N}+{ }^{10} \mathrm{~B}$ (blue dots) reactions in figure $2^{2}$. In particular, a much higher percentage of $\left(2 \alpha,{ }^{16} \mathrm{O}\right)$ events populates the less dissipative $\mathrm{Q}$-value region in the ${ }^{12} \mathrm{C}$ sample. This larger deviations are evident in the relative population of the different regions for all the even- $Z$ residues, while we observe a very good agreement in $Q_{k i n}$ distributions for the odd- $\mathrm{Z}$ residue. This difference between the two data-sets confirms a possible larger contribution of direct reactions for the ${ }^{12} \mathrm{C}+{ }^{12} \mathrm{C}$ experiment. Since a residual deviation is observed in figure 2 for $\alpha$ particles emitted in coincidence with an Oxygen in the ${ }^{14} \mathrm{~N}$ reaction, we turn now to an estimation of the possible $\alpha$ clustering effects for both reactions, both in the entrance channel and in the excited ${ }^{24} \mathrm{Mg}$.

In order to put into evidence the differences in terms of branching ratio, not only between data samples, but also considering the $\mathrm{HF} \ell$ predictions for both reactions we present, in Table 1 , the most populated channel in the experimental sample for each residue. We can see that the BR (branching ratio) of the dominant decay channels is reasonably well reproduced by the statistical model for odd $Z$ residues, while discrepancies can be seen for even- $Z$ ones.

The evaporation chains leading to a final Carbon or Oxygen or Neon residue show a preferential $\alpha$ decay in both reactions. A possible interpretation of this $\alpha$ excess could be the presence of residual $\alpha$ correlations in the excited ${ }^{24} \mathrm{Mg}$ or in its daughter nucleus ${ }^{20} \mathrm{Ne}$, populated irrespective of the entrance channel of the reaction.

\section{2 ${ }^{12} \mathrm{C}^{*}$ Hoyle state in ${ }^{24} \mathrm{Mg}^{*}$ decay: the 6- $\alpha$ channel}

We now turn to examine events where the whole available mass and charge is found as $\alpha$ particles. This selection has been performed in [4] and the inclusive results show compatibility with a sequential $\alpha$-particle emission. To improve the statistics we have analyzed here also events where only five $\alpha$ particles have been detected and extracted the properties of the sixth $\alpha$-particle from momentum and energy conservation. We have reconstructed the energy dissipated by the quantity $\mathrm{Q}_{k i n}$ previously defined $\left(\mathrm{Q}_{k i n}=\sum_{i=1}^{6} E_{i}-E_{\text {beam }}\right)$. In figure 3 (left panel) the $\mathrm{Q}_{k i n}$ distribution is shown, together with the cut used for the analysis.

In order to reconstruct the intermediate ${ }^{12} \mathrm{C}^{*}$ disassembling into 6 alphas of the ${ }^{24} \mathrm{Mg}^{*}$ formed in central collisions we have selected the three (out of six) $\alpha$-particles corresponding to the minimum

\footnotetext{
${ }^{2}$ with less dissipative we indicate events with $\mathrm{Q}_{k i n}$ greater than the neutron emission threshold
} 

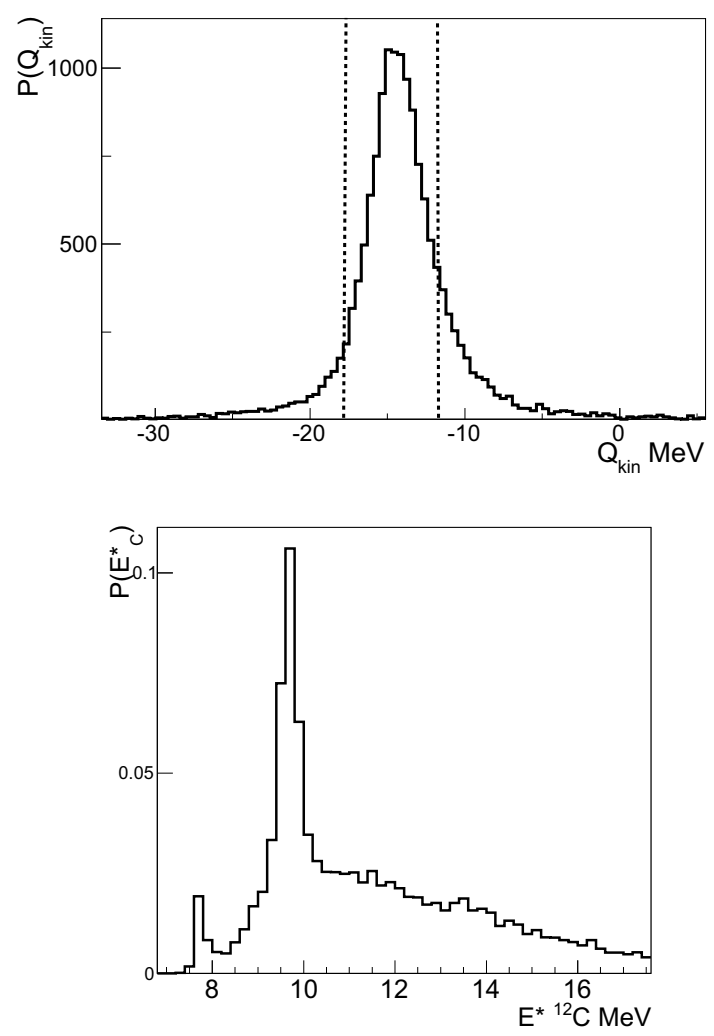

Figure 3. Upper panel: $\mathrm{Q}_{\text {kin }}$ distribution for the six $\alpha$-particles events. The vertical lines indicate the events retained for the analysis. Lower panel: Reconstructed ${ }^{12} \mathrm{C}^{*}$ excitation energy from the three $\alpha$-particles with the minimum total energy in central collisions.

${ }^{12} \mathrm{C}^{*}$ excitation energy. This is shown in figure 3 (upper panel). The two lowest ${ }^{12} \mathrm{C}^{*}$ excited states are present at 7.65 and $9.64 \mathrm{MeV}$.

The lowest energy peak in the lower panel of figure 3 corresponds to the very well known Hoyle state, which has been studied in past years by several authors $[8-11,13,14]$. The main debate consists in the interpretation of the decay of this state as sequential, via the ${ }^{8} \mathrm{Be}_{g s}$ formation, or if there is a contribution of instantaneous breakup. Different observables have been proposed to clarify this point and we will show our results for some of these observables.

The first observable is the minimum relative energy (see figure 4) of two out of the three $\alpha$-particles assigned to an excited ${ }^{12} \mathrm{C}^{*}$ produced along the ${ }^{24} \mathrm{Mg}^{*}$ decay chain. Despite of the low statistics, the results are compatible with the ones obtained for $\mathrm{HF} \ell$, indicating that the decay mainly proceeds through the intermediate formation of a ${ }^{8} \mathrm{Be}_{g s}$. The same is true for the Dalitz plot where data and $\mathrm{HF} \ell$ predictions show the same configuration (see figure 5). As usual one defines the two coordinates of the Dalitz plot as $x_{d}=\sqrt{3}\left(e_{1}-e_{2}\right)$ and $y_{d}=2 e_{3}-e_{1}-e_{2}$, where $\mathrm{e}_{i, j, k}=E_{i, j, k} /\left(E_{i}+E_{j}+E_{k}\right)$ are the $\alpha$-particle energies in the ${ }^{12} \mathrm{C}$ frame, normalized to the total energy. 


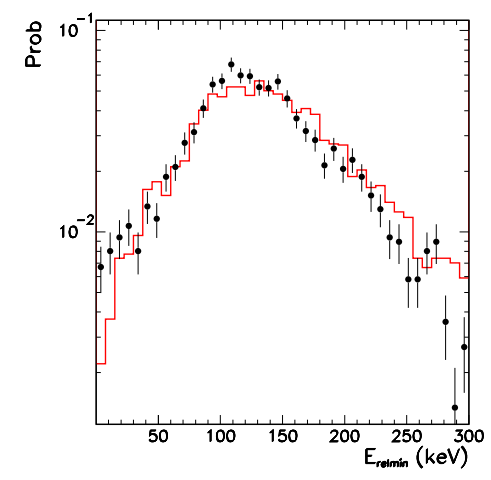

Figure 4. Central collisions: minimum relative $\alpha-\alpha$ energy (MeV). The continuous lines are the predictions of $\mathrm{HF} \ell$ predictions.
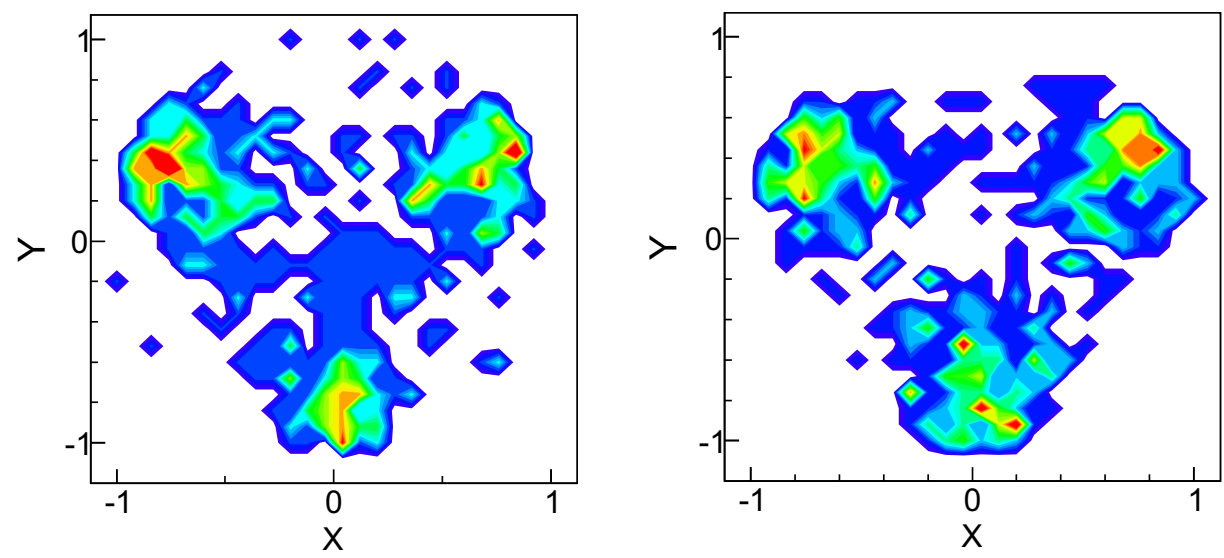

Figure 5. Central collisions. Left panel: experimental energy Dalitz plot. Right panel: HF $\ell$ Dalitz plot.

All the results for central collisions are compared to $\mathrm{HF} \ell$ predictions, showing a very good agreement. All the results obtained in inelastic channels in ${ }^{12} \mathrm{C}+{ }^{12} \mathrm{C}$ reaction [19] and in various reactions at different energies [8-12] indicate a very low limit of this contribution of the order of permil. On the other side reactions with heavier ions at higher energies, involving more complex systems, seem to be compatible with a simultaneous decay of some percent $[13,14]$. Our results for central reactions with the formation of ${ }^{12} \mathrm{C}^{*}$ Hoyle state in the decay chain of ${ }^{24} \mathrm{Mg}^{*}$, give no indications of deviations from the sequential decay mechanisms. 


\subsection{The Hoyle state in peripheral collisions}

With a different selection we repeated the same analysis performed for central collisions, with the aim of investigating the decay mechanism of the Hoyle state in different conditions. We have selected events where three $\alpha$-particles are detected in the forward cone (RCo) and nothing is detected in the rest of the solid angle. If we want to unambiguously identify "true" $\alpha$-particles we have to exploit the $\Delta$ E-E Si-CsI or the pulse shape analysis in CsI(Tl) scintillators [16]. This implies that the threshold for the identification is about $24 \mathrm{MeV}$ and consequently that the kinetic energy of ${ }^{12} \mathrm{C}$ nucleus emitting three $\alpha$-particles is relatively high. The recoil nucleus ${ }^{12} \mathrm{C}$ is therefore very slow and its energy not sufficient to reach the detector, even with a very thin target. In figure 6(left panel) the energy spectrum of the reconstructed ${ }^{12} \mathrm{C}$ quasi-projectile is shown, as calculated from the sum of the energy of the three $\alpha$-particles:

$$
E_{\text {tot }}=\sum_{i=1}^{3} E_{i}+E_{\text {rec }}
$$

where $\mathrm{E}_{\text {rec }}$ is the recoil energy of the ${ }^{12} \mathrm{C}$ quasi-target.
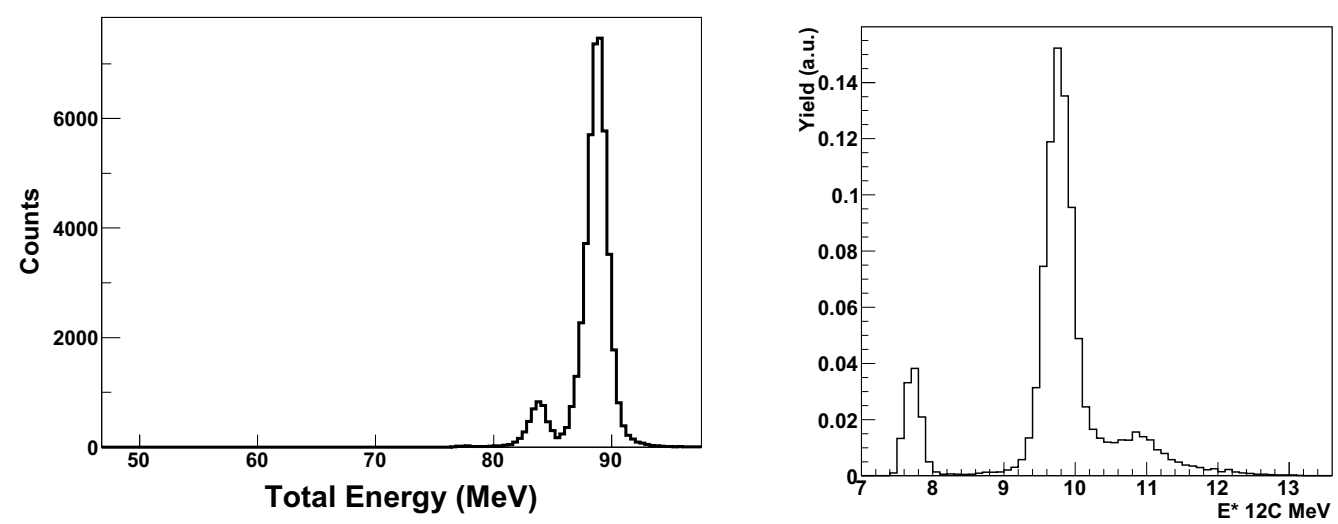

Figure 6. Total energy spectrum for "true" $\alpha$-particles (left panel). The ${ }^{12} \mathrm{C}$ excitation energy spectrum (right panel) is presented for the "true" $\alpha$-particles selecting the ${ }^{12} \mathrm{C}$ target in the ground state.

As it can be easily seen most of the kinetic energy is peaked at $\approx 88 \mathrm{MeV}$ and a small peak is present at about $84 \mathrm{MeV}$, corresponding to the ${ }^{12} \mathrm{C}$ quasi-target at about $4.4 \mathrm{MeV}$ excitation energy, the first ${ }^{12} \mathrm{C}$ excited level. The excitation energy of the ${ }^{12} \mathrm{C}$ quasi-projectile can be calculated as:

$$
E^{*}=\sum_{i=1}^{3} E_{i}-\frac{p_{C}^{2}}{2 m_{C}}+E_{t h}
$$

$E^{*}$ is presented in Figure 6 (right panel) $\left(\mathrm{E}_{t h}=-\mathrm{Q}\right)$ where $\mathrm{Q}=-7.272 \mathrm{MeV}$ is the Q-value for the decay of ${ }^{12} \mathrm{C}$ in three $\alpha$-particles. Three excited ${ }^{12} \mathrm{C}$ quasi-projectile levels are present for "true" $\alpha$ particles $(\approx 7.7,9.6$ and $10.8 \mathrm{MeV})$. We have analyzed data corresponding to the first $7.65 \mathrm{MeV}^{12} \mathrm{C}^{*}$ excited state, the first observable is the minimum relative energy of two out of the three $\alpha$-particles (figure 7). The results are compatible with the ones obtained for central collisions, indicating that the decay mainly proceeds through the intermediate formation of a ${ }^{8} \mathrm{Be}$. 


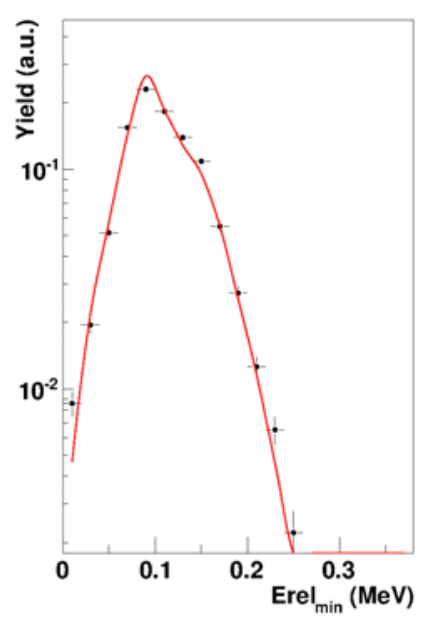

Figure 7. Peripheral collisions: minimum relative $\alpha$ - $\alpha$ energy (MeV). The continuous lines are the predictions of HF $\ell$ predictions.

The other observable proposed in the previous section is the Dalitz-plot calculated for the $\alpha$ particle energies in the ${ }^{12} \mathrm{C}$ frame, normalized to the total energy. The results from data and $\mathrm{HF} \ell$ calculations are shown in figure 8.
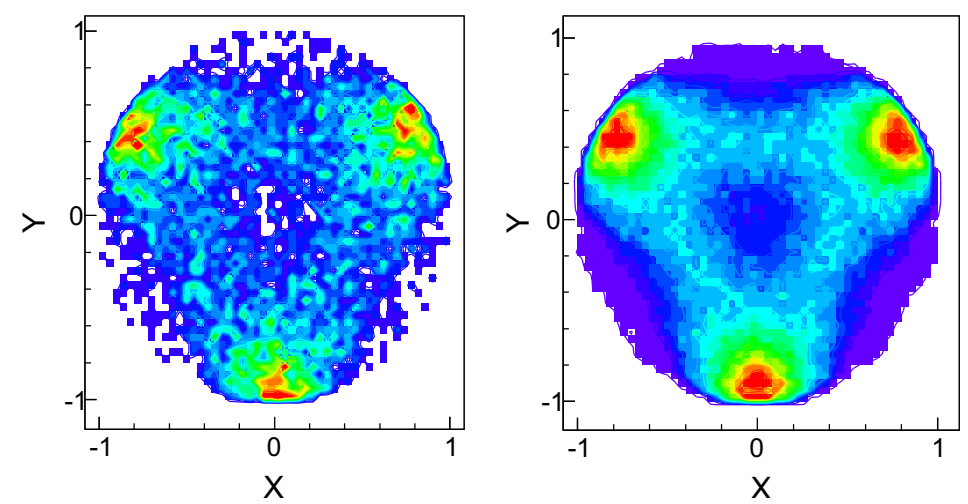

Figure 8. Peripheral collisions: Left panel: experimental energy Dalitz plot. Right panel: HF $\ell$ Dalitz plot.

We would expect that the simultaneous decay should result in three equal kinetic energies, i.e. in an enhancement of the central part of the plot which is not the case of our data. On the contrary, it shows enhancements in regions where the energy of two $\alpha$-particles are very close one to the other and the third $\alpha$-energy is far from the two others. This can be considered as a signal of the predominance of the sequential decay, as confirmed by HF $\ell$ predictions (see figure 8 right panel).

Other observables, proposed in [12] have been analyzed such as the mean energy $\left\langle E_{\alpha}>\right.$, the max- 
imum energy normalized to the total energy $\epsilon_{\max }$ and the deviation from the average center-of-mass energy, defined as $\mathrm{E}_{r m s}=\sqrt{\left\langle E_{\alpha}^{2}>-<E_{\alpha}\right\rangle^{2}}$. The results show a good agreement with HF $\ell$ predictions, i.e. with a sequential decay [20].

\subsection{The ${ }^{16} \mathrm{O}+{ }^{12} \mathrm{C}$ reaction}

For the new performed experiment ${ }^{16} \mathrm{O}+{ }^{12} \mathrm{C}$ at three different beam energies, namely $\mathrm{E}_{\text {beam }}=90,110$ and $130 \mathrm{MeV}$, the analysis has been focused up to now on the preliminary checks of collected data.
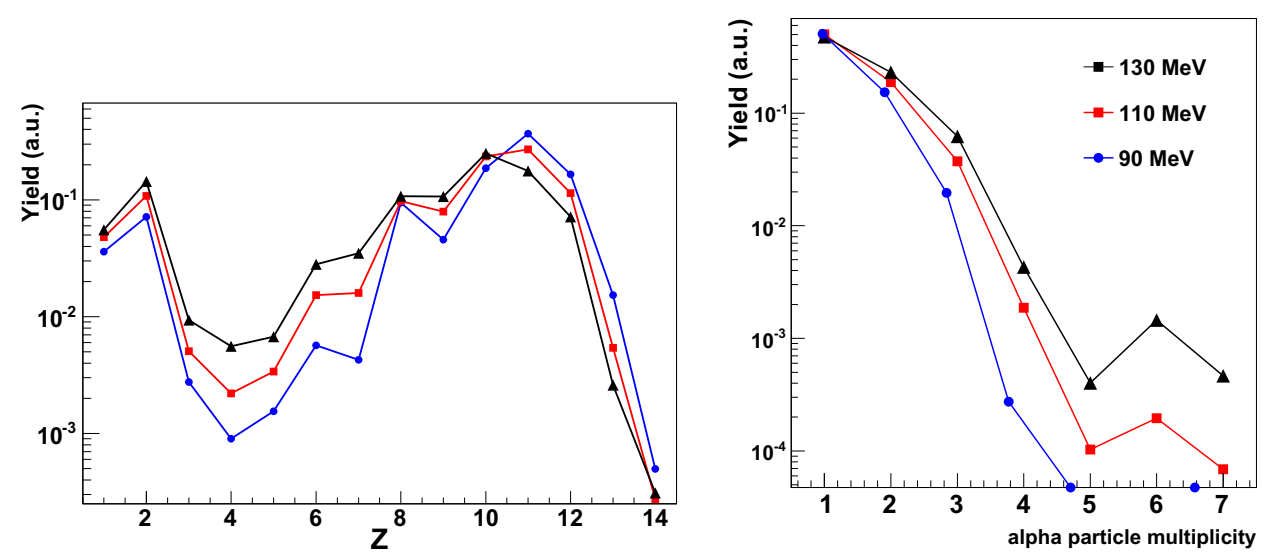

Figure 9. Preliminary results of inclusive charge distribution (left part) and alpha particle multiplicity (right part) of events selected for the reaction ${ }^{16} \mathrm{O}+{ }^{12} \mathrm{C}$ at three different beam energies.

In the case of complete fusion, such reactions lead to a fused ${ }^{28} \mathrm{Si}^{*}$ system respectively at 55 , 62 and $74 \mathrm{MeV}$ excitation energy. From our previous results on the decay of excited ${ }^{24} \mathrm{Mg}$ we expect cluster correlations to possibly persist in the ${ }^{28} \mathrm{Si}^{*}$ system, even at such high excitation energies. Extrapolating the information from Ikeda diagrams, we also expect the cluster degree of freedom be differently expressed varying the $\mathrm{E}^{*}$ of the system, thus leading to different decay patterns deviating from statistical expectations. For the three different beam energies, the charge distributions corresponding to the preliminary selection of the fusion-evaporation channel out of the entire dataset is shown in figure 9, together with the $\alpha$ multiplicity spectra detected at GARFIELD angles in coincidence with an evaporation residue in the RCo. As expected, we note a quick increase of the alpha multiplicity when the beam energy goes up.

\section{Conclusions and perspectives}

In this work we have discussed the clustering effects in nuclear reactions involving $\mathrm{N}=\mathrm{Z}$ nuclei. In particular results from the measurements with a ${ }^{12} \mathrm{C}$ beam on a ${ }^{12} \mathrm{C}$ target at $95 \mathrm{MeV}$ and the ${ }^{14} \mathrm{~N}+{ }^{10} \mathrm{~B}$ reaction at $80 \mathrm{MeV}$ beam energy are presented.

We have compared experimental data for the two reactions among them and, moreover, each experiment with the results of $\mathrm{HF} \ell$ statistical calculations for the decay of the ${ }^{24} \mathrm{Mg}^{*}$ source. The measured data are compatible with the expected behavior of a complete fusion-evaporation reaction, with some exception of specific channels corresponding to the emission $\alpha$ particles in coincidence with 
a Oxygen, Carbon or Neon residue. The experimental branching ratio excess for $\alpha$ particle emission has been quantified for both reactions, putting into evidence an effect due to the cluster nature of projectile and target in ${ }^{12} \mathrm{C}+{ }^{12} \mathrm{C}$ reaction but, at the same time, an indication of the persistence of cluster correlations also in the hot fused ${ }^{24} \mathrm{Mg}$, as suggested by data from ${ }^{14} \mathrm{~N}+{ }^{10} \mathrm{~B}$ collisions.

To investigate the importance of discrete levels and the clustering effects in the $\mathrm{CN}$ decay, we have studied the decay of the well known Hoyle state in three $\alpha$-particles. We have isolated this level in the 6-alpha decay channel in central collisions and, for the first time, we have compared its features with those of the same Hoyle level populated for the ${ }^{12} \mathrm{C}^{*}$ quasi-projectile. It has been debated if the decay mechanism is sequential, through a ${ }^{8} \mathrm{Be}_{\text {g.s. }}$. formation, or if a simultaneous decay in three $\alpha$-particles could be contribute. Our results obtained comparing peripheral and central reactions with the formation of ${ }^{12} \mathrm{C}^{*}$ Hoyle state in the decay chain of ${ }^{24} \mathrm{Mg}^{*}$, give no indications of deviations from the sequential decay mechanisms. Further measurements are needed, increasing the energy and the number of nucleons involved, in order to investigate if in medium effects may distort the decay of the Hoyle state.

A natural extension of the same experimental scheme will be to compare the decay of a compound system formed in collisions between stable $\alpha$-clustered reaction partners, or induced by an unstable light projectile on a chosen target in the future SPES facility at Legnaro National Laboratory. Together with the previously studied ${ }^{24} \mathrm{Mg}$, the compound nucleus ${ }^{28} \mathrm{Si}$ can be formed with light radioactive beams provided by SPES.

\section{References}

[1] W. Von Oertzen 2010 Clusters in Nuclei, Lecture Notes in Physics 818 109-127.

[2] K. Ikeda, Prog. Theor. Phys. (Suppl.) extra number, 464 (1968).

[3] G. Baiocco et al., Phys. Rev. C 87,(2013) 054614.

[4] L. Morelli et al., J. Phys. G: Nucl. Part. Phys. 41 (2014) 075107.

[5] Y. Alhassid, G. F. Bertsch Phys. Rev. C 68 (2013) 044322;

[6] R. B. Wiringa, Phys. Rev. C 62 (2000) 014001.

[7] F. Hoyle The Astrophysical Journal, Supplement Series 1954112.

[8] M. Freer et al. PR 107 (1994) R1751.

[9] J. Manfredi et al. PRC 85 (2012) 037603.

[10] O. S. Kirsebom et al. PRL 108 (2012) 202501.

[11] T. K. Rana et al. PRC 88 (2013) 021601.

[12] M. Itoh M et al. PRL 113 (2014) 102501.

[13] Ad. R. Raduta et al. PLB 705 (2011) 65.

[14] L. Quattrocchi et al. 2015 Contribution to Nucleus nucleus 2015 NN2015, Catania, Italy.

[15] M. Bruno et al., Eur. Phys. Journ. A 49 (2013) 128.

[16] G. Pasquali et al., Nucl. Instr. And Meth., A 570 (2007) 126.

[17] M. E. Ortiz et al Phys. Rev. C 25 (1982) 1436.

[18] L. Morelli et al., J. Phys. G: Nucl. Part. Phys. 41 (2014) 075108.

[19] L. Morelli et al., annual report 2014 LNL.

[20] L. Morelli et al 2014 J. Phys. G: Nucl. Part. Phys. to be published. 